\title{
Asymmetric Rosen-Zener-like transition through a soliton-surface-plasmon photonic Josephson junction with spatially varying coupling
}

\author{
Güneş Aydındoğan* and Kaan Güven \\ Department of Physics, Koç University, Istanbul 34450, Turkey
}

(Received 24 July 2017; published 1 November 2017)

\begin{abstract}
The transition dynamics of photons between an optical soliton in a nonlinear dielectric waveguide and a spatially coupled surface-plasmon excitation on a parallel flat metal surface can be formulated in analogy to that of a Josephson junction of two-level (double-well) Bose-Einstein condensates, albeit with a nonlinear coupling that inherently depends on the population imbalance of the levels. The present work demonstrates that asymmetric Rosen-Zener-like transitions can be obtained through this optical Josephson junction, by turning on and off the coupling across a hyperbolically varying separation between the soliton and the surface-plasmon. The transitions can generate full population transfer, population splitting, or merging between the quasistationary initial and final states, which are defined by a fixed population imbalance in the decoupled limit. Transitions from a pure soliton or pure surface-plasmon initial state are found to be robust against the relative phase, whereas the transitions from an initial state with mixed population depend strongly on the relative phase. The soliton-surface-plasmon system also bears similarities to the spatially coupled optical waveguides which are introduced further as the classical analogs of the spatial adiabatic passage and stimulated Raman adiabatic passage mechanisms in quantum and atom optics.
\end{abstract}

DOI: 10.1103/PhysRevA.96.053802

\section{INTRODUCTION}

Two-level systems are ubiquitous in physics. The transition dynamics between levels through implicit or explicit coupling resides at the roots of atomic and quantum physics [1-4] and applies to many other physical systems through analogies. A recent addition to the two-level system repository has been the coupled optical soliton-surface-plasmon system which is defined by a nonlinear dielectric channel and a parallel metal surface, that are separated by a linear dielectric layer. Several models have been proposed to describe the dynamics of the system, which range from a heuristic model [5] subject to the near-resonance conditions with a symmetric coupling, to a rigorous treatment of the Maxwell's equations with an asymmetric coupling [6,7]. In the heuristic model, the dynamics of the soliton and surface-plasmon field amplitudes is formulated as a coupled nonlinear-linear oscillator system where the coupling depends inherently to the soliton amplitude. This dependence brought the inquiry whether adiabatic or diabatic transitions between the soliton-like and surface-plasmon-like eigenmodes can be controlled by the soliton amplitude, but remained undisclosed thus far [5].

A follow-up study [8] revealed that the heuristic model can be formulated as a photonic Josephson junction, in analogy to that of a Bose-Einstein condensate (BEC) in a double well [9-11]. This potentially enables the projection of various properties of the well-known BEC Josephson junctions to the relatively unexplored soliton-surface-plasmon Josephson junction. In particular, the adiabatic or diabatic transitions in double- or triple-well BEC systems were already investigated extensively with Landau-Zener (LZ) [12-14], Rosen-Zener (RZ) [14] schemes, with their generalized form [15], and with various temporal modulations of the barrier potential $[16,17]$. Hence, one objective of the present paper is to generate

\footnotetext{
*gaydindogan@ku.edu.tr
}

LZ- or RZ-like transition mechanisms for the soliton-surfaceplasmon Josephson junction.

To this end, we can also highlight the similarity of the present work with the studies of adiabatic passage in coupled optical waveguide systems [18-21] which are, in turn, introduced as classical optic analogies of quantum adiabatic dynamics of atomic levels that are coupled and driven by light. Primarily known as the rapid adiabatic passage (RAP) [22,23] and stimulated Raman adiabatic passage (STIRAP) $[23,24]$, these mechanisms found diverse implementations in atomic and molecular optics, cavity quantum electrodynamics, cold atom systems, and classical wave optics. We refer the reader to the extensive reviews by Vitanov et al. [25] and Menchon-Enrich et al. [26] and references therein on this subject. As a note, the standard STIRAP mechanism is based on three resonantly coupled (atomic) levels, but a two-level analogy involving a temporally varying coupling detuning has been established [27]. In the case of waveguide couplers, a spatial instead of a temporal detuning occurs by bending the waveguide axes [20,21].

With this setting, the present paper investigates the transition dynamics of the coupled soliton-surface-plasmon Josephson junction, by introducing a spatial detuning to the coupling. We will focus on the transition of quasistationary states which are defined by fixed respective populations of the soliton and surface-plasmon states in the decoupled limit. Evidently, the pure soliton (or surface-plasmon) quasistationary states turn out to be the eigenstates of this two-level system, in the absence of coupling. We demonstrate complete transfer of photons between the quasistationary initial and final pure states of the soliton and surface-plasmon, as well as tailored population splitting or merging. The dependence of the coupling to the soliton amplitude (i.e., to the relative population of the levels) manifests itself with an asymmetry in the transition characteristics, which resembles remarkably to that of the asymmetric Rosen-Zener transition. We ought to note that strictly adiabatic transitions are not the subject of the present 
work. The analogy to the aforementioned adiabatic LZ- or RZ transitions intends to provide a provisional perspective.

The paper is organized as follows: Sec. II reviews briefly the soliton-surface-plasmon Josephson junction (SSPJJ) model and describes its extension with spatially tuned coupling. The Rosen-Zener dynamics in double-well BEC, and the spatial adiabatic passage in coupled optical waveguides, are highlighted for comparison. Section III presents the transition between quasistationary states as a function of the model parameters and the spatial parameters of the coupling. Section IV details particular cases such as the transition between pure states and equally populated states. The dependence of the transitions on the relative phase of the initial quasistationary state is discussed. The transitions are illustrated on the phase space.

\section{MODEL AND FORMULATION}

We begin by a brief description of the coupled solitonsurface-plasmon model introduced in Ref. [5], and of its formulation as a Josephson junction given in Ref. [8]. The surface-plasmon resides on a metal-linear dielectric interface along the $z$ axis and the soliton propagates within a Kerrtype nonlinear dielectric channel embedded in the dielectric, running parallel to interface at a constant distance in the $+x$ direction. The model is two dimensional ( $z-x$ plane) and all spatial variables are scaled by the free-space wave vector $k$. The quasistatic case is given by an ansatz which expresses the total electric field by the superposition of the soliton and surface-plasmon electric fields:

$$
\Psi(z, x)=c_{p}(z) \psi_{p}(x)+c_{s}(z) \psi_{s}\left(x,\left|c_{s}\right|\right),
$$

where $c_{p, s}$ are the longitudinal field amplitudes and $\psi_{s}=$ $\operatorname{sech}\left[\sqrt{2\left(\beta_{s}-1\right)}(x-d)\right], \psi_{p}=\exp \left(-x \sqrt{\beta_{p}^{2}-1}\right)$ are the lateral evanescent field profiles of the soliton and surfaceplasmon, respectively. $d$ (scaled as $k d$ ) is the distance between the soliton propagation axis and the metal interface. $\beta_{s} \equiv$ $1+\gamma\left|c_{s}\right|^{2} / 4$ and $\beta_{p} \equiv k_{p} / k$ denote the respective propagation parameters of the soliton and surface-plasmon. $\gamma$ is the nonlinearity parameter of the dielectric and $k_{p}$ is the surfaceplasmon wave vector.

In the quasistatic case, the field amplitudes change along the propagation axis and thus the dynamics is formulated as a function of $z$. The overlap of the lateral field profiles generates the coupling, which is a function of the soliton's longitudinal field amplitude due to the self-focusing property of the nonlinear dielectric. Under weak coupling, the longitudinal field amplitudes are governed by a linear oscillator (surfaceplasmon) and nonlinear oscillator (the soliton) coupled equation system as follows [5]:

$$
\left(\begin{array}{l}
\ddot{c_{p}} \\
\ddot{c_{s}}
\end{array}\right)=\left(\begin{array}{cc}
-\beta_{p}^{2} & q \\
q & -\beta_{s}^{2}
\end{array}\right)\left(\begin{array}{l}
c_{p} \\
c_{s}
\end{array}\right) .
$$

Here $\ddot{c}_{s, p} \equiv \frac{\partial^{2} c_{s, p}}{\partial z^{2}}$. The explicit form of the coupling $q$ will be discussed further below. In this work, we study symmetric coupling only. The extension of the aforementioned model to nonsymmetric coupling was reported in recent studies in which the soliton to plasmon coupling is larger than the opposite [6]. We stress that both $\beta_{s}$ and $q$ depends on the soliton amplitude $\left|c_{s}\right|$. The phase-matching condition $\beta_{s}=\beta_{p}$ gives rise to a mechanism of resonant energy transfer between the soliton and the surface-plasmon [5].

Equation (2) is reduced to first order by substituting $c_{p, s}=C_{p, s}(z) e^{i z}$ and employing the slowly varying amplitude approximation, where only the first order derivatives of the complex amplitudes $C_{p, s}(z)$ are retained:

$$
-i\left(\begin{array}{l}
\dot{C}_{p} \\
\dot{C}_{s}
\end{array}\right)=\left(\begin{array}{cc}
\beta_{p}-1 & -q / 2 \\
-q / 2 & \beta_{s}-1
\end{array}\right)\left(\begin{array}{l}
C_{p} \\
C_{s}
\end{array}\right) .
$$

The Josephson junction formulation is then obtained by writing the complex amplitudes as $C_{s, p}(z)=A_{s, p}(z) e^{i \phi_{s, p}(z)}$ and by expressing Eq. (3) in terms of the fractional population imbalance $Z=\frac{\left|A_{s}\right|^{2}-\left|A_{p}\right|^{2}}{N}$, and the relative phase parameter $\phi=\phi_{s}-\phi_{p}$. The system is assumed to be lossless, in which the total field amplitude (i.e., photon number) is constant and normalized: $N \equiv\left|A_{s}\right|^{2}+\left|A_{p}\right|^{2}=1$. To reveal the BJJ analogy, two parameters are defined:

$$
\begin{gathered}
\Lambda \equiv \frac{\gamma N}{8}, \\
\Delta E \equiv \Lambda-\left(\beta_{p}-1\right) .
\end{gathered}
$$

The soliton-surface-plasmon Josephson junction (SSPJJ) dynamics is then given by [8]

$$
\begin{gathered}
\dot{Z}=-q(Z) \sqrt{1-Z^{2}} \sin \phi, \\
\dot{\phi}=\Lambda Z+q(Z) \frac{Z}{\sqrt{1-Z^{2}}} \cos \phi+\Delta E .
\end{gathered}
$$

Reference [28] suggested the following functional form of $q$ :

$$
q(Z)=\sqrt{\frac{1+Z}{2}} e^{-d \sqrt{2 \Lambda(1+Z)}} .
$$

Near the strong soliton limit $(Z \approx 1)$, the lateral profile of the soliton is narrow due to self-focusing effect and the coupling varies exponentially: $q \sim \exp \left(-d \sqrt{\gamma / 2}\left|A_{s}\right|\right)$. As the soliton amplitude decreases, the soliton's lateral profile widens. Thus, the coupling increases and reaches maximum near the strong surface-plasmon limit $(Z \approx-1)$ before decreasing again.

The present work adopts this form of the coupling and elaborates on introducing a spatial dependence to it, such that it is turned on and off asymptotically along the course of propagation. For this purpose, we suppose that the soliton propagates along a hyperbolic trajectory depicted in Fig. 1 and formulated as

$$
x^{2}-\alpha^{2} z^{2}=d^{2}, \quad x \geqslant 0, \quad z \in(-\infty, \infty),
$$

where $d$ stands now for the minimum coupling distance and $\alpha=\tan \theta$ is the slope of the asymptote. The modified coupling function then reads

$$
q(z, Z)=\sqrt{\frac{1+Z}{2}} e^{-\sqrt{d^{2}+\alpha^{2} z^{2}} \sqrt{2 \Lambda(1+Z)}},
$$

where $d$ determines the maximum value attainable by the coupling and $\alpha$ defines a logarithmic switching rate of the coupling along the course of propagation. For $\alpha<1$ and $d$ sufficiently large, the paraxial approximation is applicable and the dynamics can still be described by Eqs. (6) and (7) with $q$ given in Eq. (10). 


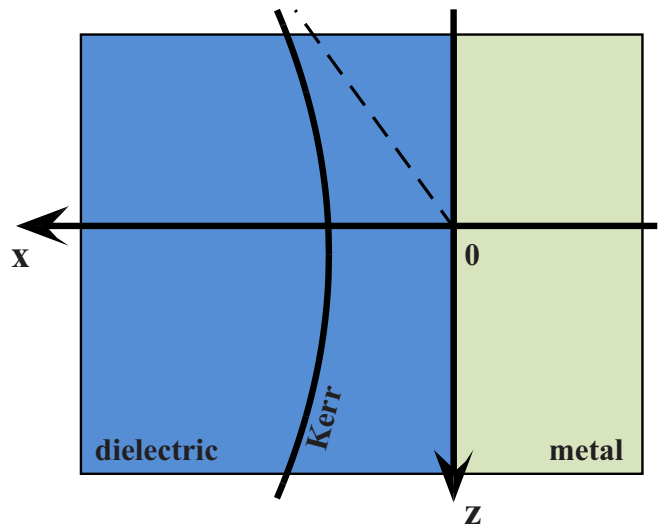

FIG. 1. Schematic of the soliton-surface-plasmon model with a spatially tuned coupling. The soliton propagates in a Kerr-type nonlinear dielectric channel that follows a hyperbolic path with respect to the metal dielectric interface located on the $z$ axis.

To this end, a comparison of the SSPJJ [Eqs. (6) and (7)] with BJJ systems is illustrative. Smerzi et al. formulated the BJJ of double-well Bose-Einstein condensates with constant coupling between the wells $[9,10]$. Here we quote the equations for the case with time-dependent detuning and coupling parameters studied by Nesterenko et al. [15]:

$$
\text { (BJJ) } \dot{Z}=-\bar{\Omega}(t) \sqrt{1-Z^{2}} \sin \phi,
$$

$$
\dot{\phi}=\frac{1}{2}\left[\Lambda Z+\bar{\Omega}(t) \frac{Z}{\sqrt{1-Z^{2}}} \cos \phi+\Delta E(t)\right]
$$

In these equations, the coupling is formulated as $\Omega(t)=$ $K \bar{\Omega}(t)$, where $K$ is the amplitude. The parameters $\Lambda=\frac{U N}{2 K}$ and $\Delta E=\frac{E_{1}(t)-E_{2}(t)}{2 K}$ describe, respectively, the interaction of bosons in the same well and the detuning between the wells. The derivatives are with respect to scaled time $t \rightarrow 2 \mathrm{~K} t$. The work employs a linear detuning rate in a finite time interval $\left[\Delta E(t)=\alpha t,-t_{s}<t<t_{s}\right]$, and a Gaussian-shaped coupling $\bar{\Omega}(t)=\exp \left\{-\frac{(\bar{t}-t)^{2}}{2 \Gamma^{2}}\right\}$, where $\bar{t}$ is the center and $\Gamma$ is the width of the pulse. This system leads to some generalization of the Landau-Zener and Rosen-Zener type adiabatic transition mechanisms in BEC systems.

While the similarity of equation pairs (6)-(7) and (11)-(12) encourages the analogy, important differences ought to be pointed out. In BJJ, $\Lambda$ can be positive or negative corresponding to repulsive or attractive nonlinear interaction of atoms in a well, whereas its counterpart in SSPJJ model is positive definite. Reference [15] reported that the adiabatic transitions with full population transfer in the BJJ occur for attractive $(\Lambda<0)$ interaction, and that the repulsive interaction breaks the adiabatic transport. The second difference is the dynamic (static) nature of $\Delta E$ in BJJ (SSPJJ). We remind the reader that the stationary states $\left(Z^{*}, \phi^{*}\right)$ associated with the diabatic to adiabatic transitions are determined from Eqs. (6) and (7) as

$$
\begin{gathered}
\dot{Z}=0 \rightarrow \phi^{*}=n \pi, \quad n: \text { integer, } \\
\dot{\phi}=0 \rightarrow \Lambda Z^{*} \pm q \frac{Z^{*}}{\sqrt{1-\left(Z^{*}\right)^{2}}}+\Delta E=0,
\end{gathered}
$$

where the \pm sign in Eq. (14) is for the even (odd) choice of $n$ in Eq. (13). In the absence of coupling ( $q=0)$, a typical "level crossing scheme" for the diabatic states would require a detuning rate for $\Delta E$, similar to that given in Eq. (12). The very definition of $\Delta E$ in SSPJJ makes it difficult to conceive a mechanism for this purpose as either the nonlinear dielectric or the plasmon wave vector has to be tunable along the course of propagation. Henceforth, we will focus on the transitions between the quasistationary states in the SSPJJ which are discussed in the next section. It is worth mentioning that the SSPJJ bears a strong resemblance to the optical waveguides with a spatially detuned coupling. In Ref. [20] a curved optical directional coupler is discussed which employs a spatial complex hyperbolic secant scheme to modify both the coupling and the mismatch of the propagation constants along the course of propagation, to generate a shortcut to adiabatic passage (SAP) [29] between the waveguide channels. The scheme is based on the Allen-Eberly transition scheme for two-level atoms [22]. Another work [21] presents the Raman adiabatic passage-like transfer of light between a straight and a curved waveguide where the propagation constants and the coupling are again modified spatially. Since our present work deals with the transitions between quasistationary states (see Sec. III) we do not carry on the analogy further here. Adiabatic transitions of the SSPJJ under spatially modulated coupling will be discussed elsewhere.

\section{TRANSITION DYNAMICS}

The quasistationary states are described by a constant population imbalance $Z_{ \pm \infty}$ and a constant phase rate $\dot{\phi}_{ \pm \infty}$ in the decoupled limit,

$$
\lim _{z \rightarrow \pm \infty} \dot{Z}=0, \quad \lim _{z \rightarrow \pm \infty} \dot{\phi}=\Lambda Z_{ \pm \infty}+\Delta E .
$$

The transition between initial and final quasistationary states $(Z, \dot{\phi})_{-\infty} \rightarrow(Z, \dot{\phi})_{+\infty}$ is then computed by integrating Eqs. (6) and (7) with Eq. (10) in a finite range that ensures the limiting behavior given above at its boundaries. For brevity, the quasistationary states are called simply states in the rest of the paper.

Figure 2(a) presents a showcase that full transition between the pure states can be achieved by the hyperbolic modulation of the coupling. In this particular case, the transition is reversible, but asymmetric under $Z \rightarrow-Z$. The asymmetric dependence of the coupling to the population imbalance generates this unique behavior. Remarkably, the coupling profile shown in Fig. 2(b) resembles that of the asymmetrized Rosen-Zener model [30], which is an exactly solvable two-state atom model with an asymmetric secant-hyperbolic shaped excitation pulse $\Omega(t)$ and constant detuning $\Delta E$ between the states.

For a general analysis, the transition outcome $\left(Z_{+\infty}\right)$ from initial pure states $\left(Z_{ \pm \infty}\right)$ is calculated as a function of the modulation parameters $(d, \alpha)$. This is depicted in Fig. 3. In producing this figure, the minimum value of $d$ and maximum value of $\alpha$ are chosen to be consistent with the weak coupling and the paraxial approximations. For the top panels, the initial pure state is soliton $\left(Z_{-\infty}=1\right)$ indicated by the white color. The bottom panels are obtained by starting with a pure surface-plasmon state $Z_{-\infty}=-1$, indicated by the black 
(a)
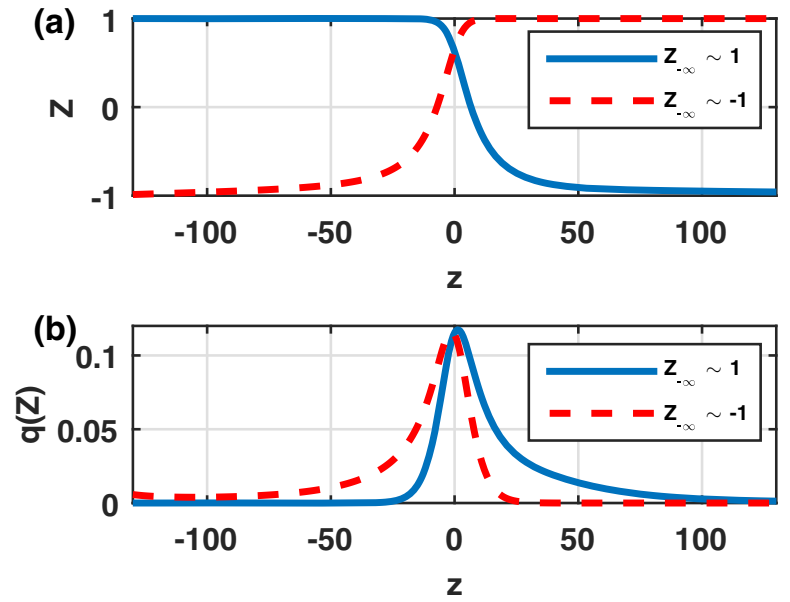

FIG. 2. (a) The full transition starting with pure soliton (solid blue curve) and pure surface-plasmon (broken red curve) states. (b) The asymmetric variation of the coupling during the respective transitions. The model parameters are $\Lambda=0.04, \Delta E=0.02$. The spatial modulation parameters are $\alpha=0.63, d=5.70$.

color. Thus, the darker (brighter) streaks in the top (bottom) panels indicate a transition dominantly to the other state.

Visual comparison of the respective top and bottom panels show that the asymmetry of the coupling in $Z$ renders the transition spectra different for $Z_{-\infty}=1$ and $Z_{-\infty}=-1$ initial pure states. The transition outcome exhibits rapid variations in the lower left part of the spectra where both $d$ and $\alpha$ are small (i.e., both the strength and the duration of the coupling are relatively large), which is expected.

The nonlinearity parameter $\Lambda$ increases across the panels from left to right while $\beta_{p}=1.02$ is kept constant. This exemplifies negative, zero, and positive settings of the detuning parameter $\Delta E$. The negative (positive) value of $\Delta E$ defines a detuning in favor of the surface-plasmon (soliton) state. We observe that the transition spectrum under a surface-plasmon favorable detuning [Figs. 3(a) and 3(d)] is more sensitive to the modulation parameters. The spectra becomes subdued under soliton favorable detuning [Figs. 3(c) and 3(f)]. In this case, the pure soliton initial state does not couple to the surface-plasmon state at all for $d>10$, indicated by the broad white region in Fig. 3(c). This is expected, since $\Delta E>0$ implies strong nonlinearity and the enhanced self-focusing of the soliton prevents an effective coupling to the surface-plasmon. On the other hand, the pure surface-plasmon initial state is transferred to soliton-dominant states as shown by the broad light-colored region of Fig. 3(f).

These transition spectra show that an initial pure state can be distributed with arbitrary population imbalance among the two levels, by changing the spatial modulation parameters $(d, \alpha)$. For each column of Fig. 3, where the model parameters $(\Delta E, \Lambda)$ are fixed, one can specify values of $(d, \alpha)$ to generate certain transition characteristics in the same system. For instance, the small red dots in Figs. 3(c) and 3(f) indicate the modulation parameters used in Fig. 2 that yield the full population transfer between the pure states. The values $d \approx 5, \alpha \approx 0.6$ in Figs. 3(a) and 3(d) exemplify another response in which both initial pure states are transferred to the surface-plasmon pure state.

\section{DISCUSSION}

\section{A. Transitions between pure and equally populated states}

This subsection focuses on the transitions between the pure $(Z= \pm 1)$ and equally populated $(Z=0)$ states. Figures $4(\mathrm{a})$ and 4 (b) show population splitting $\left(Z_{-\infty}= \pm 1 \rightarrow Z_{\infty}=0\right)$ and Figs. 4(c) and 4(d) show population merging $\left(Z_{-\infty}=0 \rightarrow\right.$ $Z_{\infty}= \pm 1$ ). While these transitions are obtained under various settings of the system parameters (indicated in the legend box), it is possible to generate the transitions just by changing the spatial modulation parameters [for instance, for Figs. 4(c) and 4(d) only $d$ changes].
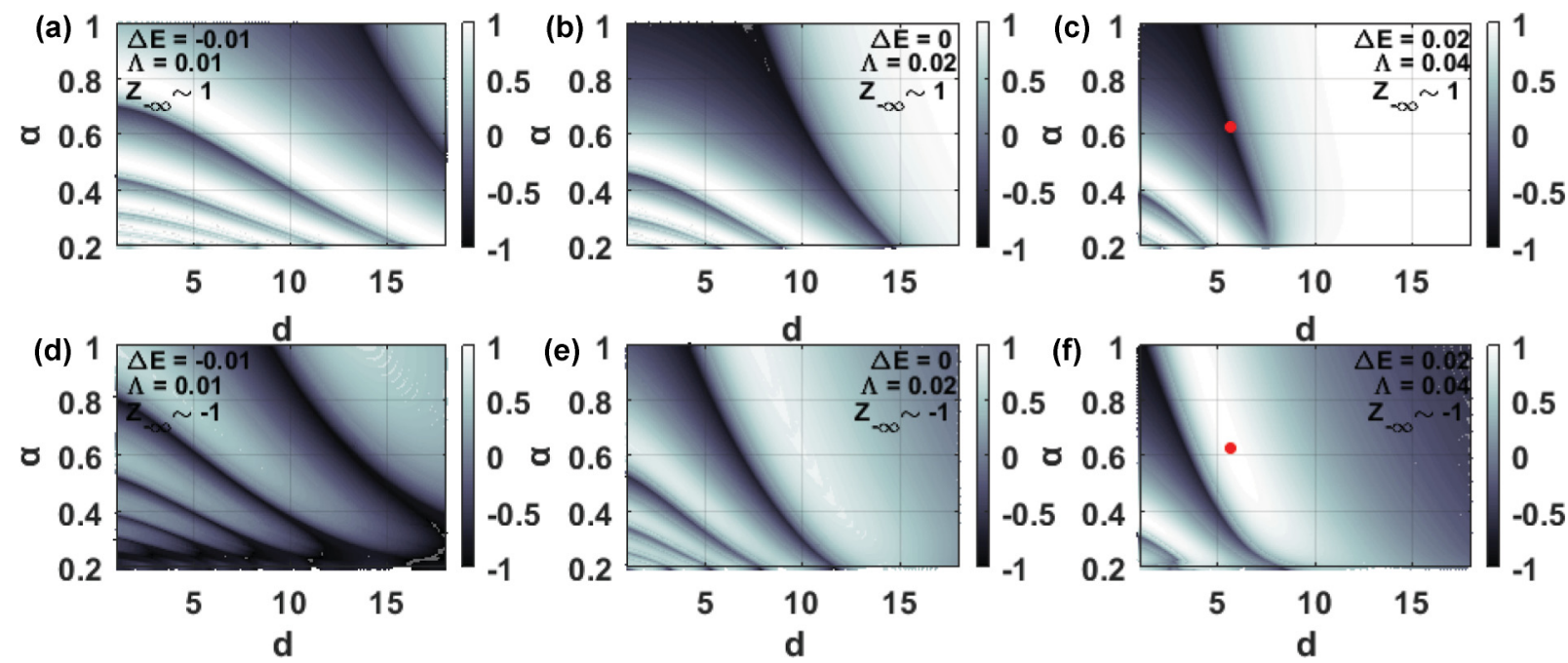

FIG. 3. The color coded spectrum of the final state $Z_{\infty}$ in the modulation parameter space $d$ and $\alpha$. The top (bottom) panels correspond to an initial state with $Z_{-\infty}=1(-1)$. The darker (brighter) streaks in the top (bottom) panels indicate a transition dominantly to the other state. The detuning $\Delta E$ increases across the panels from left to right, which is achieved by increasing $\Lambda$ under constant $\beta_{p}$. The red dots in (c) and (f) indicate the modulation parameters of Fig. 2, giving full transition. 


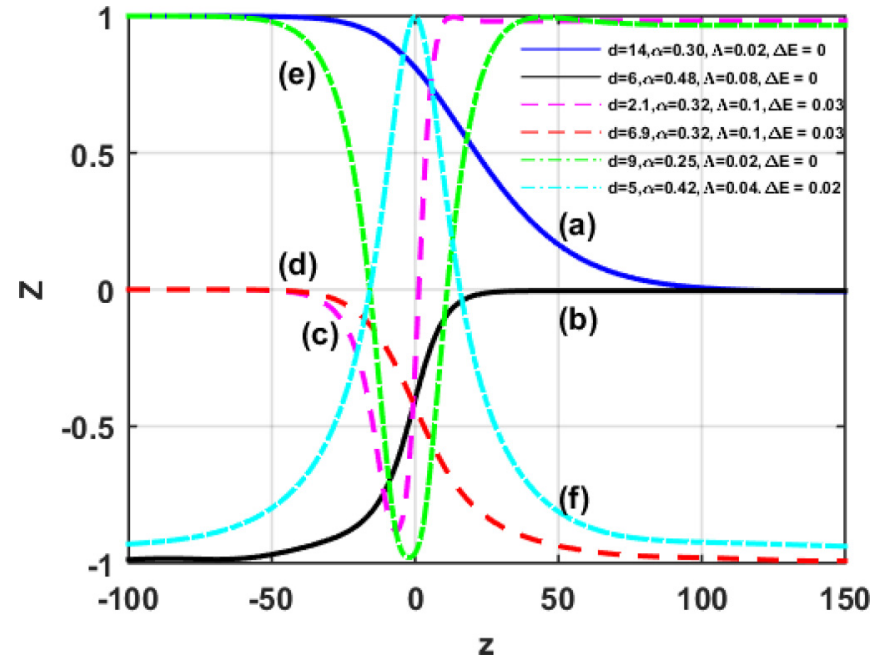

FIG. 4. The curves (a)-(d) show transitions between pure $(Z=$ $\pm 1)$ and equally populated $(Z=0)$ states obtained by spatial modulation. The curves (e) and (f) indicate, respectively, a transition in which a spatially localized surface-plasmon or soliton state is generated.

\section{B. Transition induced surface-plasmon or soliton hotspot}

For particular values of $d, \alpha$, complete population transfer from and to the same pure state can occur successively around $z=0$. This generates a spatially localized soliton or surface-plasmon state [Figs. 4(e) and 4(f)]. It might be interesting to investigate these within a three-level system, where the localized level mediates an interaction between the two propagating levels [31].

Figure 5 visualizes the normalized intensity distribution of the soliton and surface-plasmon fields that is calculated using Eq. (1), in correspondence to some transitions given in Figs. 2 and 4: Full population transfer [Figs. 5(a) and 5(b)], population splitting [Fig. 5(c)], merging [Fig. 5(d)], and localized states of surface-plasmon [Fig. 5(e)] or soliton [Fig. 5(f)]. Note that the asymmetry of the coupling range affects the extend of localization for the surface-plasmon and soliton hotspots, where the former is more confined compared to the latter.

\section{Phase dynamics}

Up to here, the initial relative phase $\left(\phi_{-\infty}\right)$ is taken to be zero. However, since the quasistationary initial states in Eq. (13) are defined up to an arbitrary phase, the effect of nonzero values of $\phi_{-\infty}$ on the transition outcome $Z_{\infty}$ needs to be investigated. Figure 6 shows this effect for pure initial states (red and blue curves), and for equally populated initial state (dotted curve). In Fig. 6(a) the modulation parameters $d, \alpha$ are tuned to the full transition setting, with values as given in Fig 2. Figure 6(b) uses different values.

The flat running curves in both Figs. 6(a) and 6(b) indicate that the transition outcome $Z_{\infty}$ from pure initial states $Z_{-\infty}= \pm 1$ is rather insensitive to the initial phase. In contrast, the transition outcome from $Z_{-\infty}=0$ initial state exhibits a strong, nonlinear phase dependence. As seen by the dotted curves, the features of this dependence are specific to the particular settings of the model parameters
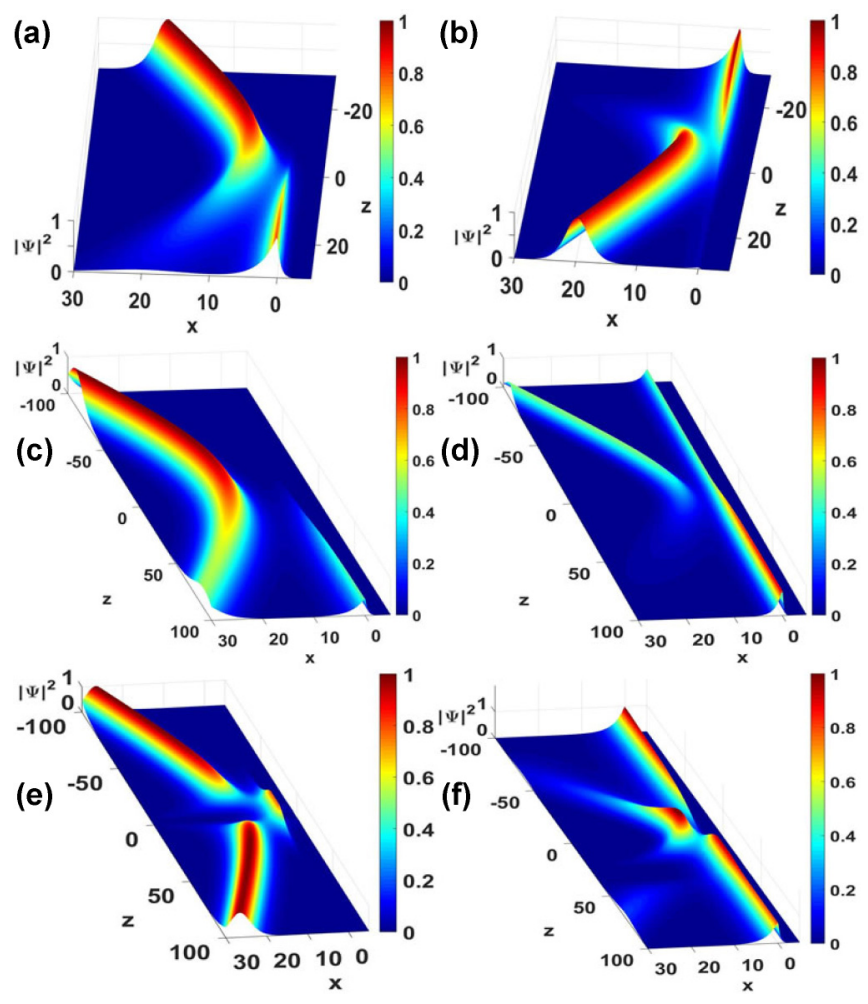

FIG. 5. The normalized intensity distribution of the soliton and surface-plasmon fields [Eq. (1)] for the transition $Z_{-\infty} \rightarrow Z_{\infty}$ as (a) $1 \rightarrow-1$, (b) $-1 \rightarrow 1$, (c) $1 \rightarrow 0$, and (d) $0 \rightarrow-1$. These correspond to Fig. 2(a) and Figs. 4(b) and 4(c), respectively. The bottom panels (e) and (f) show the transitions corresponding to those in Figs. 4(e) and 4(f). Panels are drawn at different isometric viewing angles to enhance the visibility.

$(\Lambda, \Delta E)$ and the modulation parameters $(d, \alpha)$. If the initial phase were controllable, it would be possible to generate a
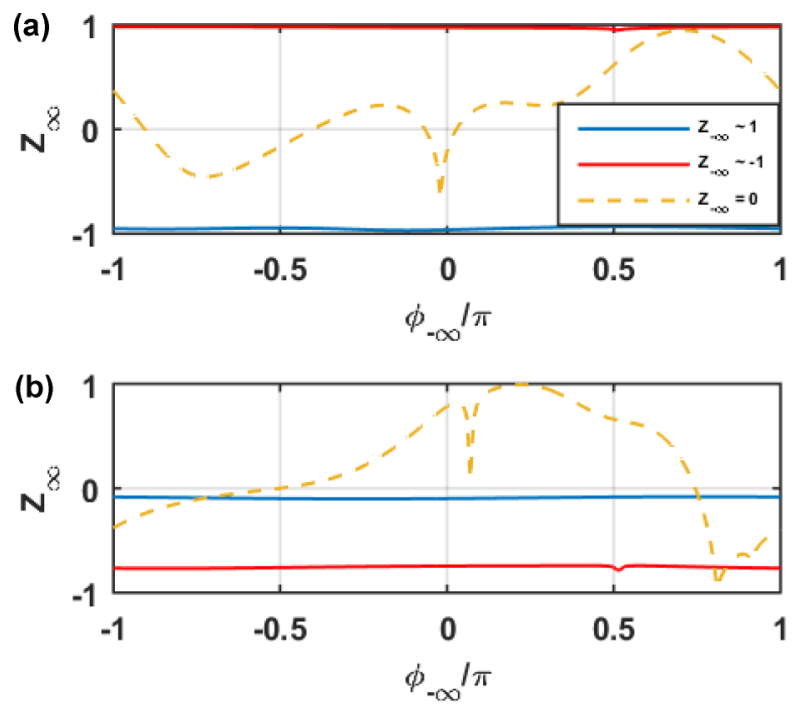

FIG. 6. The transition outcome $Z_{\infty}$ as a function of initial phase $\phi_{-\infty}$ for the pure initial states (red and blue curves), and for the equally populated initial state (dotted curves). In (a), the modulation parameters are tuned to the full transition setting: $d=5.70, \alpha=0.63$. In (b), different values of $d=5.60, \alpha=0.4$ are used. In both cases, $\Lambda=0.04$ and $\Delta E=0.02$. 


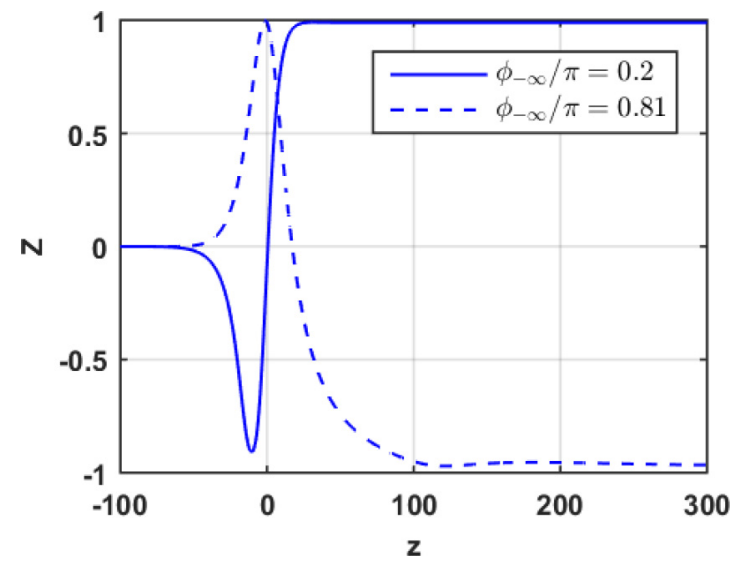

FIG. 7. Phase controlled population transfer from the $Z_{-\infty}=0$ state to either pure states. $\phi_{-\infty}=0.20 \pi(0.81 \pi)$ generates the transition $Z 0 \rightarrow 1(-1)$. The parameters $\Lambda, \Delta E, d, \alpha$ are the same as in Fig. 6(b).

phase controlled output profile for the population imbalance. This is illustrated in Fig. 7 which, by using the modulation parameters of Fig. 6(b), shows that $\phi_{-\infty}=0.20 \pi$ yields $Z_{-\infty}=0 \rightarrow Z_{\infty}=1$, whereas $\phi_{-\infty}=0.81 \pi$ gives $Z_{-\infty}=$ $0 \rightarrow Z_{-\infty}=-1$. Interestingly, these transitions generate a hotspot of a pure state before being transferred to the other pure state. The origin of this behavior become clear in the phase-space description of the transitions, which are discussed in the next subsection.

Figures 8(a)-8(c) present the phase propagation dynamics for a set of initial relative phase values in the range $[-\pi, \pi]$ and with the initial state $Z_{-\infty}=-1,0$, and 1 , respectively. This figure is complementary to Fig. 6, where the respective final states $Z_{\infty}$ are plotted.

The phase level curves of the quasistationary states that are governed by Eq. (15) comprise parallel running lines. This is clearly observed in the far left of Figs. 8(b) and 8(c). In Fig. 8(a) they remain further to the left of the propagation axis. In Fig. 8(c) the level curves corresponding to different initial phases split, coalesce, and get pinned around odd multiples of $\pi / 2$ while approaching the maximum coupling point $(z=0)$. After that, the coalesced level curves disperse slightly, which we shall explain later through the phase-space description shown in Fig. 9.

Figure 8(a) shows a different behavior: the level curves branch and coalesce quite early during propagation. The reason relates to the different magnitude of the second term in Eq. (7) near $Z_{\infty}=1$ and $Z_{\infty}=-1$, respectively. By taking the explicit form of $q(Z)$ in Eq. (8) into account, we see that the second term can be significant for $Z \approx-1$, even at $z<<0$, due to the vanishingly small exponent. In contrast, it is exponentially small for $Z \approx 1$ at $z<<0$. After $z=0$, the level curves in Fig. 8(a) make another branching and finally propagate in parallel. All branchings start and end at odd multiples of $\pi / 2$.

The coalescent behavior of phase level curves in Figs. 8(a) and 8 (c) explains why the transitions from pure initial states $\left(Z_{\infty}= \pm 1\right)$ are robust against different initial phases as seen in Fig. 6. In contrast, the phase dynamics of the $Z=0$ initial state [Fig. 8(b)] has a complicated behavior through the transition region around $z=0$ : the level curves end up with different slopes (i.e., phase rates) which indicate different values of $Z_{\infty}$.

\section{Phase space description}

The transition phenomena investigated thus far can be visualized in the phase space of the system. This amounts for tracking a state point (the initial quasistationary state) as it moves on the continuously changing landscape of the phase space. Figures 9 and 10 illustrate the transitions between the pure states shown in Fig. 2(a), and the transitions from the equal population state shown in Fig. 7, respectively.

The panels of Fig. 9 from left to right show the snapshots of the phase space at various values of $z$ in the range $[-100,100]$. The filled (empty) symbols show the present (past) location of the state. Dotted lines are added for guidance. The quasistationary states are located on the open trajectories given by the horizontal running lines. During the transition, several critical points emerge at integer multiples of $\pi$, that shift vertically, and disappear in the phase space. They alter the phase landscape
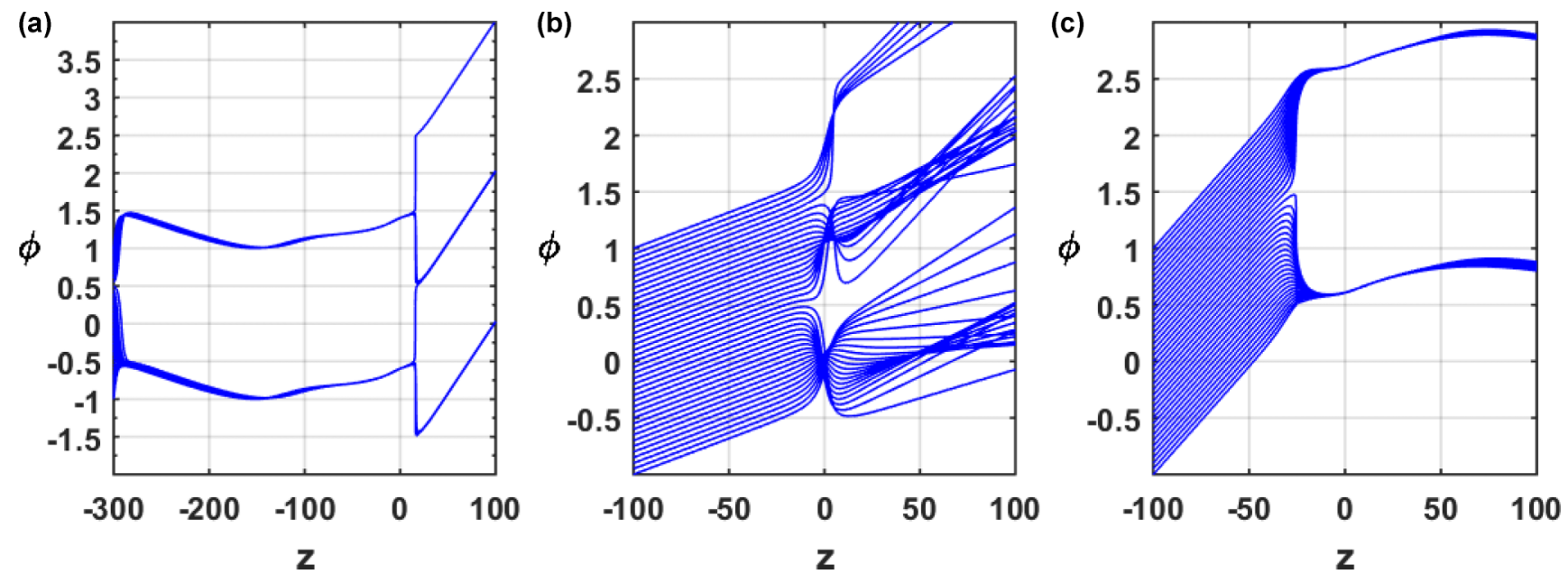

FIG. 8. Propagation of the phase level curves with initial values in the range $-\pi$ and $\pi$, and for the initial population state (a) $Z_{-\infty}=-1$, (b) $Z_{-\infty}=0$, and (c) $Z_{-\infty}=1$, respectively. The parameters $\Lambda, \Delta E, d, \alpha$ are the same as in Fig. 6(a). 


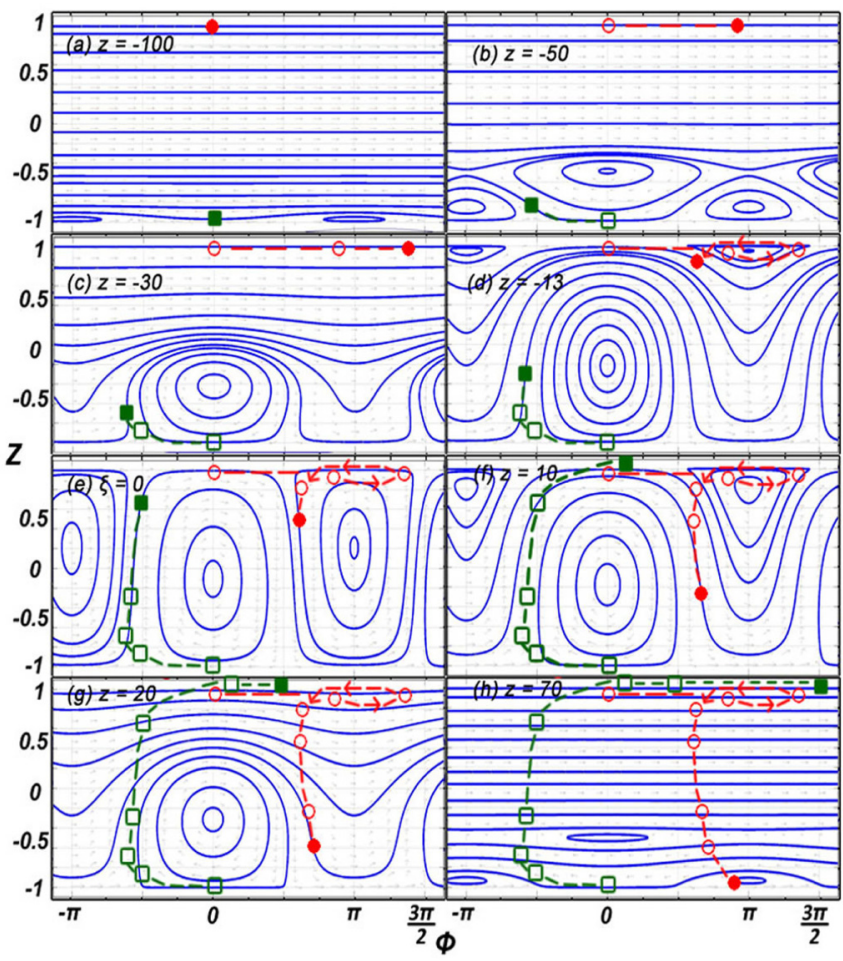

FIG. 9. Phase-space snapshots at various $z$ values showing the propagation of the quasistationary initial states $Z_{-\infty}=1$ (solid red circle) and $Z_{-\infty}=-1$ (solid green square), which generate full population transfer. The initial phase is set to zero for both states. Blank symbols and dashed lines show earlier locations and trajectory for guidance. The parameters $\Lambda, \Delta E, d, \alpha$ are the same as in Fig. 2.

substantially by creating closed orbits. The moving state point gets trapped around the critical points and cross the phase space vertically to generate a population transfer. Note that the vertical movement occurs near the odd multiples of $\pi / 2$ which define a sharp boundary between oppositely running closed orbits. This explains the branching and coalescence of the phase level curves shown in Figs. 8(a) and 8(c).

Regarding the slight dispersion of the phase level curves in Fig. 8(c) for $z>50$, we note in Fig. 9(h) that the state point gets trapped in a closed orbit at the vicinity of an emerged critical point. Due to the small but finite extend of this closed orbit, the final $Z_{\infty}$ also shows a small variation and causing the observed dispersion.

Figure 10 shows how the initial quasistationary $Z=0$ state with different initial phases ends up in different pure states, complementary to Fig. 7 . The transition $Z_{-\infty}=0 \rightarrow Z_{\infty}=1$ is completed at $z=30$ [Fig. 7(g)], whereas the transition $Z_{-\infty}=0 \rightarrow Z_{\infty}=-1$ requires a much longer propagation range to reach the quasistationary state [Fig. 7(h)].

Several features of the phase space can be highlighted:

(1) The asymmetry of the coupling range for solitondominant and surface-plasmon-dominant states: For $|z|>50$ the state trajectories are mostly flat for $Z>0$ and more dynamic for $Z<0$ with significant population exchange.

(2) Emerging critical points enable population transition: Under spatially varying coupling, critical points emerge at integer multiples of $\pi$ and the closed orbits around them

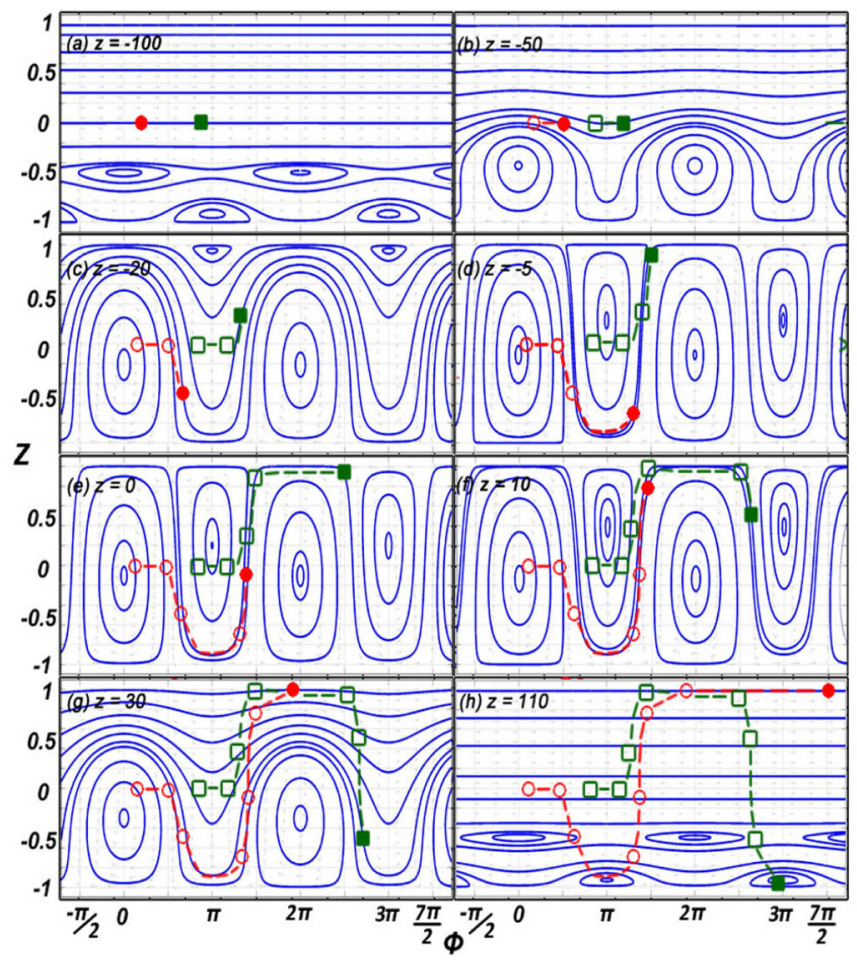

FIG. 10. Phase-space snapshots at various $z$ values showing the propagation of the quasistationary initial state $Z_{-\infty}=0$, with initial phase of $\phi=0.20 \pi$ (solid red circle) and $\phi=0.81 \pi$ (solid green square). Blank symbols and dashed lines show earlier locations and trajectory for guidance. The parameters $\Lambda, \Delta E, d, \alpha$ are the same as in Fig. 6(b).

expand to cover the entire phase space with increasing coupling. This enables the state point to move vertically in the phase space and hence the population transfer.

(3) Phase boundaries at odd multiples of $\pi / 2$ : Sharp phase boundaries are formed at odd multiples of $\pi / 2$ between the outer closed orbits of critical points, while the system propagates through $z=0$. These phase boundaries split and coalesce different initial phase curves into a single curve as shown in Fig. 8.

We must add as a final note that adding a dissipation mechanism would alter the phase space significantly [28] and hence the transition dynamics.

\section{CONCLUSION}

This paper investigated the dynamics of population transition among the quasistationary states of a soliton-surfaceplasmon Josephson junction with a spatially modulated coupling. Tailored population transfer (including full inversion, splitting, merging of population) can be achieved by adjusting the modulation parameters. The transition dynamics is similar to the asymmetric Rosen-Zener transition in double-well BoseEinstein condensates with a temporally modulated coupling. A similarity can also be drawn with the transfer of light between spatially coupled waveguides.

The experimental feasibility of realizing the solitonsurface-plasmon interaction has already been addressed in the literature. Bliokh et al. gave a practical assessment of 
the resonant soliton-surface-plasmon interaction at a goldchalcogenide glass interface in the far infrared regime (wavelength $\lambda \sim 5 \mu \mathrm{m}$ ), with realistic parameters [5]. Another recent study considered a planar structure that is composed of chalcogenide glass coated with silica and gold films, and emphasized a low power $\left(\sim 1 \mathrm{GW} / \mathrm{cm}^{2}\right)$ requirement to generate a coupled soliton-surface-plasmon excitation [32]. Steady-state planar optical soliton excitation in indium phosphide doped with iron was reported under low excitation intensity $\left(50 \mathrm{~mW} / \mathrm{cm}^{2}\right)$ and applied dc field $(9 \mathrm{kV} / \mathrm{cm})$ [33]. This semiconductor structure is particularly attractive for monolithic integration to optoelectronic components operating at telecommunication wavelengths. Motivated by these studies, the soliton-surfaceplasmon Josephson junction can be a prospective component with unique features for nonlinear optic and plasmonic applications.

\section{ACKNOWLEDGMENTS}

K.G. wishes to acknowledge the support of the Turkish Academy of Sciences Associate Member Research Grant and of the Scientific and Technological Research Council of Turkey Project No. 111T285.
[1] L. D. Landau, Phys. Z. Sowjet. 2, 46 (1932).

[2] C. Zener, Proc. R. Soc. London Ser. A 137, 696 (1932).

[3] N. Rosen and C. Zener, Phys. Rev. 40, 502 (1932).

[4] I. I. Rabi, Phys. Rev. 51, 652 (1937).

[5] K. Y. Bliokh, Y. P. Bliokh, and A. Ferrando, Phys. Rev. A 79, 041803 (2009).

[6] C. Milián, D. E. Ceballos-Herrera, D. V. Skryabin, and A. Ferrando, Opt. Lett. 37, 4221 (2012).

[7] A. Ferrando, C. Milián, and D. V. Skryabin, J. Opt. Soc. Am. B 30, 2507 (2013).

[8] Y. Ekşioǵlu, Ö. E. Müstecaplioǵlu, and K. Güven, Phys. Rev. A 84, 033805 (2011).

[9] A. Smerzi, S. Fantoni, S. Giovanazzi, and S. R. Shenoy, Phys. Rev. Lett. 79, 4950 (1997).

[10] S. Raghavan, A. Smerzi, S. Fantoni, and S. R. Shenoy, Phys. Rev. A 59, 620 (1999).

[11] S. Giovanazzi, A. Smerzi, and S. Fantoni, Phys. Rev. Lett. 84, 4521 (2000).

[12] J. Liu, L. Fu, B.-Y. Ou, S.-G. Chen, D. I. Choi, B. Wu, and Q. Niu, Phys. Rev. A 66, 023404 (2002).

[13] D. Witthaut, E. M. Graefe, and H. J. Korsch, Phys. Rev. A 73, 063609 (2006).

[14] D.-F. Ye, L.-B. Fu, and J. Liu, Phys. Rev. A 77, 013402 (2008).

[15] V. O. Nesterenko, A. N. Novikov, A. Yu Cherny, F. F. de S. Cruz, and E. Suraud, J. Phys. B: At. Mol. Opt. Phys. 42, 235303 (2009).

[16] C. Weiss and T. Jinasundera, Phys. Rev. A 72, 053626 (2005).

[17] Q. Zhang, P. Hanggi, and J. B. Gong, Phys. Rev. A 77, 053607 (2008).
[18] S. Longhi, Phys. Rev. E 73, 026607 (2006).

[19] S. Longhi, G. D. Valle, M. Ornigotti, and P. Laporta, Phys. Rev. B 76, 201101(R) (2007).

[20] K. Paul and A. K. Sarma, Phys. Rev. A 91, 053406 (2015).

[21] H. Oukraou, L. Vittadello, V. Coda, C. Ciret, M. Alonzo, A. A. Rangelov, N. V. Vitanov, and G. Montemezzani, Phys. Rev. A 95, 023811 (2017).

[22] L. Allen and J. H. Eberly, Optical Resonance and Two-Level Atoms (Dover, New York, 1987).

[23] N. V. Vitanov, T. Halfmann, B. W. Shore, and K. Bergmann, Ann. Rev. Phys. Chem. 52, 763 (2001)

[24] M. P. Fewell, B. W. Shore, and K. Bergmann, Aust. J. Phys. 50, 281 (1997).

[25] N. V. Vitanov, A. A. Rangelov, B. W. Shore, and K. Bergmann, Rev. Mod. Phys. 89, 015006 (2017).

[26] R. Menchon-Enrich, A. Benseny, V. Ahufinger, and A. D. Greentree, Rep. Prog. Phys. 79, 074401 (2016)

[27] N. V. Vitanov and B. W. Shore, Phys. Rev. A 73, 053402 (2006).

[28] Y. Ekşioǵlu, Ö. E. Müstecaplioǵlu, and K. Güven, Phys. Rev. A 87, 023823 (2013).

[29] X. Chen, I. Lizuain, A. Ruschhaupt, D. Guéry-Odelin, and J. G. Muga, Phys. Rev. Lett. 105, 123003 (2010).

[30] N. V. Vitanov, J. Phys. B: At. Mol. Opt. Phys. 27, 1351 (1994).

[31] K. Güven and G. Aydindoğan (unpublished).

[32] W. Walasik, V. Nazabal, M. Chauvet, Y. Kartashov, and G. Renversez, Opt. Lett. 37, 4579 (2012); W. Walasik, G. Renversez, and Y. V. Kartashov, Phys. Rev. A 89, 023816 (2014).

[33] M. Chauvet, S. A. Hawkins, G. J. Salamo, M. Segev, D. F. Bliss, and G. Bryant, Opt. Lett. 21, 1333 (1996). 